\title{
Influence of the fsr locus on biofilm formation by Enterococcus faecalis lacking gelE
}

Although enterococci are normal commensals of the gastrointestinal tract of humans and many mammals, they have emerged as important pathogens causing nosocomial infections, including urinary tract, bloodstream, wound and surgical-site infections, in addition to their

long-recognized importance as a cause of infective endocarditis (Murray, 1990). The binding of Enterococcus faecalis to heart valves and to various biomaterials (Joyanes et al., 1999; Toledo-Arana et al., 2001; Mohamed et al., 2004; Seno et al., 2005) and medical devices (Keane et al., 1994; Dautle et al., 2003; Sandoe et al., 2003) is the presumed initiating factor that then allows subsequent formation of a biofilm. Biofilm formation in E. faecalis has been reported to be influenced by various genes such as esp, initially reported by Toledo-Arana et al. (2001). Gelatinase, which strongly influences virulence in models of peritonitis, endocarditis (Singh et al., 1998, 2005), endophthalmitis (Engelbert et al., 2004) and in vitro translocation (Zeng et al., 2005), has been reported to influence biofilm formation (Mohamed et al., 2003, 2004; Hancock \& Perego, 2004; Kristich et al., 2004). Other E. faecalis genes such as epa, atn (Mohamed et al., 2004), bop (Hufnagel et al., 2004), salA and salB (Mohamed et al., 2006) have also been shown to influence biofilms. Biofilm production has been shown to be regulated by quorum-sensing systems in several important pathogens, including $f_{s} r$ in $E$. faecalis, which was shown to have a pronounced effect on biofilms (Hancock \& Perego, 2004; Mohamed et al., 2003, 2004; Pillai et al., 2004).

The fsr locus, a homologue of the staphylococcal agr loci, global regulators of virulence and metabolism (Dunman et al., 2001), positively regulates the expression of gelatinase and serine protease in E. faecalis (Qin et al., 2000) and autoregulates expression of the $f_{s r} B$ and $f_{s r} C$ genes (Nakayama et al., 2001; Qin et al., 2001). Most strains of E. faecalis are $\mathrm{gelE}^{+}$, although only about $60 \%$ of them have the $f_{s} r$ locus and are gelatinase producers by standard assay (Roberts et al., 2004). We have shown recently that all three $f s r$ mutants (phenotypically $\mathrm{GelE}^{-} \mathrm{SprE}^{-}$by standard assay; Qin et al., 2000; Singh et al., 2005), as well as gelE mutants (GelE $\mathrm{SprE}^{-}$), showed decreased biofilm production (Mohamed et al., 2003, 2004), a finding confirmed by Hancock \& Perego (2004); the latter also showed that the introduction of $f s r A B C$ and $f s r A B C /$ gelE/ sprE into strain FA2-2 $\left(\mathrm{GelE}^{-}\right.$, with point mutations in the $f s r B$ and $f_{s r} C$ loci) resulted in gelatinase production and increased biofilm production (Hancock \& Perego, 2004). However, our initial results had also suggested that $f_{s} r$ may have a role, in addition to activating gelatinase-mediated biofilm formation, as the $f_{s} r$ mutants formed slightly more biofilm than the gelatinase/serine protease double mutant TX5128 (Mohamed et al., 2004). In order to study the effect of $f_{s} r$ on biofilm formation by E. faecalis, independent of activation of its gelatinase production, we used E. faecalis clinical isolates lacking gelE and $f s r$ as hosts in this study.

Table 1. Strains and plasmids used in this study

\begin{tabular}{|c|c|c|}
\hline Strain/plasmid & Characteristics/origin & Reference \\
\hline $\mathrm{JH} 2-2$ & $\begin{array}{l}\text { Well-characterized laboratory strain; plasmid-free; } g e l E^{+} \text {, } \\
\text { lacks } f_{s} A, f_{s r} \text {; GelE }\end{array}$ & Jacob \& Hobbs (1974) \\
\hline TX5441.01 & $\mathrm{JH} 2-2(\mathrm{pAT} 18) ; \mathrm{Em}^{\mathrm{r}}$ & This study \\
\hline TX5441 & JH2-2(pTEX5249); GelE ${ }^{+}$, Em $^{\mathrm{r}}$ & This study \\
\hline TX5453.01 & TX0006(pAT18); Em $^{\mathrm{r}}$ & This study \\
\hline TX5453 & TX0006(pTEX5249); Em ${ }^{\mathrm{r}}$ & This study \\
\hline TX0014 & $\begin{array}{l}\text { Endocarditis isolate; strong biofilm producer; } \\
\text { lacks } f \text { srB, gelE; GelE }\end{array}$ & Mohamed et al. (2004) \\
\hline TX5454.01 & TX0014(pAT18); Em ${ }^{\mathrm{r}}$ & This study \\
\hline
\end{tabular}


The bacterial strains and plasmids used in this study are listed in Table 1. For biofilm experiments, bacteria were first grown overnight in tryptic soy broth plus $0 \cdot 25 \%$ glucose (TSBG) with or without erythromycin, as appropriate, and then in TSBG for biofilm formation. Hosts lacking $f s r$ and gelE that produced strong, medium and weak biofilms from our culture collections and JH2-2 were used as recipients. pTEX5249 (pAT18 carrying $\left.f_{s} A B C\right)$ and its appropriate control, pAT18, were introduced by electroporation and plated on Todd-Hewitt (TH) agar with $25 \mu \mathrm{g}$ erythromycin $\mathrm{ml}^{-1}$.

Erythromycin-resistant colonies carrying pTEX5249 were identified by PCR and mini-plasmid preparations. Gelatinase production was tested at $72 \mathrm{~h}$ on $\mathrm{TH}$ agar plates containing 3\% gelatin (Qin et al., 2000). Biofilm formation and adherence to a polystyrene surface were assessed quantitatively as well as by phase-contrast microscopy, as described previously (Mohamed et al., 2004).
We first introduced pTEX5249 into JH2-2 (esp ${ }^{-}$gelE $^{+}$, but gelatinase-negative due to the absence of most of the $f s r$ locus with truncated $f_{s r} \mathrm{C}$ and a $23.9 \mathrm{~kb}$ deletion; Nallapareddy et al., 2005) and found gelatinase production and increased biofilm production $(53 \%)$ compared with the respective controls, similar to results reported by Hancock \& Perego (2004) with FA2-2. We then tested the strong biofilm producer TX0014 (biofilm $\mathrm{OD}_{570}>2 \cdot 0$ ) (lacking $f_{s} r$, gelE and esp) after the introduction of pTEX5249 (TX5454) and found that biofilm formation was significantly reduced ( $41 \% ; P<0 \cdot 0001$, Student's $t$-test) compared with the wild-type and control TX5454.01 (TX0014 containing pAT18) (Fig. 1a). Similarly, when we tested the medium biofilm producer TX0006 (biofilm $\mathrm{OD}_{570}$ of 1-2), after the introduction of $f$ s $r$ genes, resulting in TX5453, this strain also showed a significant decrease $(29 \% ; P<0 \cdot 0001$, Student's $t$-test) in biofilm formation relative to its respective controls, TX0006
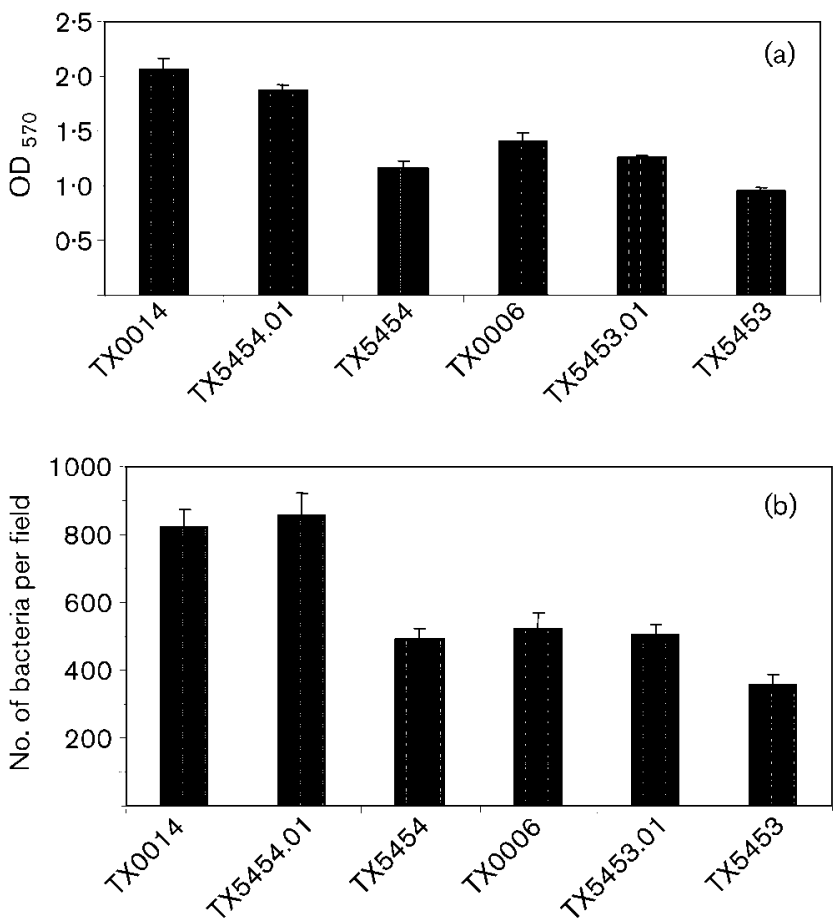

Fig. 1. (a) Biofilm formation by $f s r$-lacking and fsr-containing hosts at $24 \mathrm{~h}$. The experiment was performed three times in quadruplicate and the $\mathrm{OD}_{570}$ (mean $\pm S D$ ) is shown. (b) Initial attachment to a polystyrene surface. The experiment was performed twice and five fields were counted in each experiment. The number of bacteria per field (mean $\pm S D)$ is shown.

and TX5453.01 (Fig. 1a). By phase-contrast microscopy, the $f s r$-containing strains displayed either no evidence of (TX5453) or fewer (TX5454) microcolonies with empty areas of plastic surface and reduced biofilm production (Fig. 2). TX5453 and TX5454 harbouring the $f s r$ locus also showed less initial attachment than $f s r$-lacking strains (Fig. $1 \mathrm{~b}$ ), indicating that $f s r$ negatively controls initial adherence, a prerequisite of biofilm formation. We also tested a weak biofilm producer, TX0238, but were unable to recover pTEX5249-containing derivatives from it; none of our other $f_{s r} /$ gelE-lacking strains produced weak biofilm.

Under some conditions, agr mutants of Staphylococcus aureus (Vuong et al., 2000) and Staphylococcus epidermidis (Vuong et al., 2003) have been shown previously to display enhanced biofilm formation and increased adhesion to epithelial cells (Vuong et al., 2004) compared with a corresponding isogenic agr wild-type, indicating that agr can suppress biofilm formation. Our results suggest that $f_{s} r$ has an effect, independent of proteases, on biofilm formation in E. faecalis and that this effect is in the same direction as that of $a g r$ of staphylococci. In summary, the introduction of the $f s r$ locus into gelElacking strains resulted in reduced initial attachment, no microcolonies and/or fewer clusters of cells and a reduced amount of biofilm production relative to the parental strains, indicating that the $f s r$ locus has an additional role, independent of gelatinase production, that leads to decreased initial attachment followed by reduced biofilm production in E. faecalis.

\section{Acknowledgements}

This work was supported by NIH grant R37 AI47923 from the Division of Microbiology and Infectious Diseases, NIAID, to B. E. M.

Jamal A. Mohamed ${ }^{1,2}$ and Barbara E. Murray ${ }^{1,2,3}$

Division of Infectious Diseases, Department of Internal Medicine", Center for the Study of Emerging and Reemerging Pathogens ${ }^{2}$ and Department of Microbiology and Molecular Genetics, University of Texas Medical School, Houston, TX 77030, USA

Correspondence: Barbara E. Murray (bem.asst@uth.tmc.edu) 
(a)

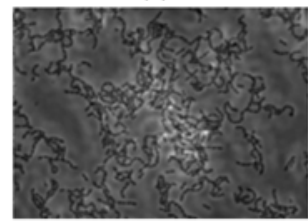

$12 \mathrm{~h}$

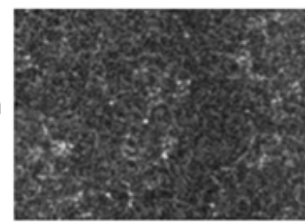

$24 \mathrm{~h}$

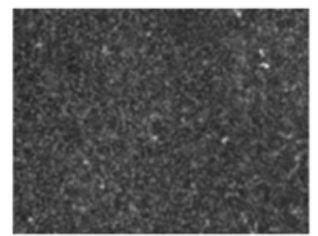

(d)
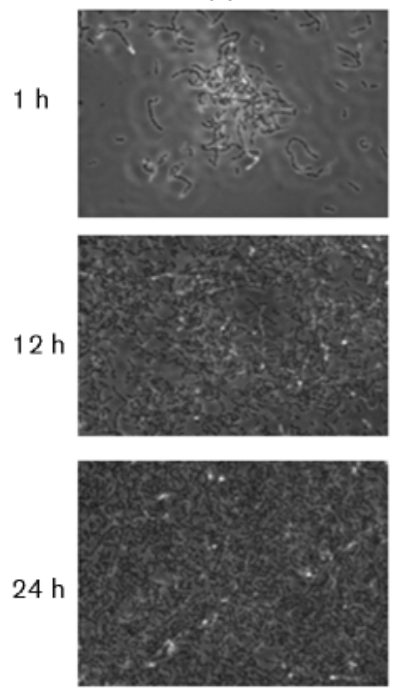

(b)
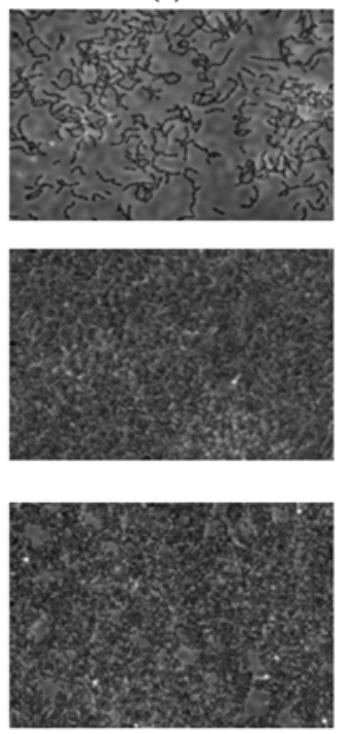

(e)
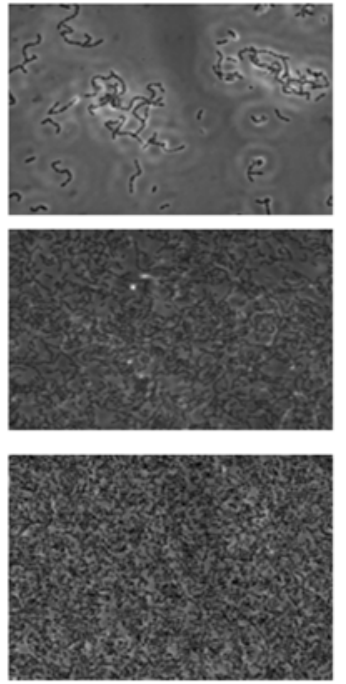

(c)
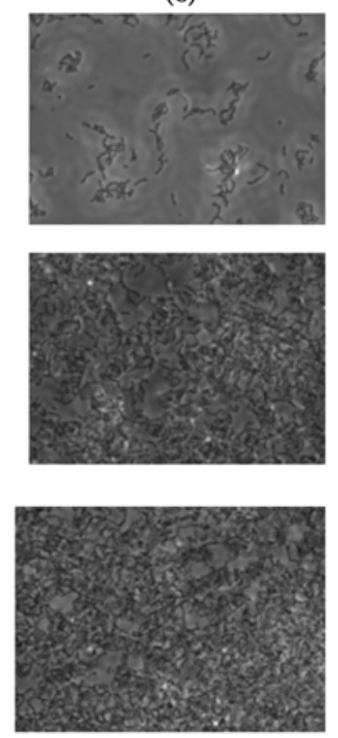

(f)
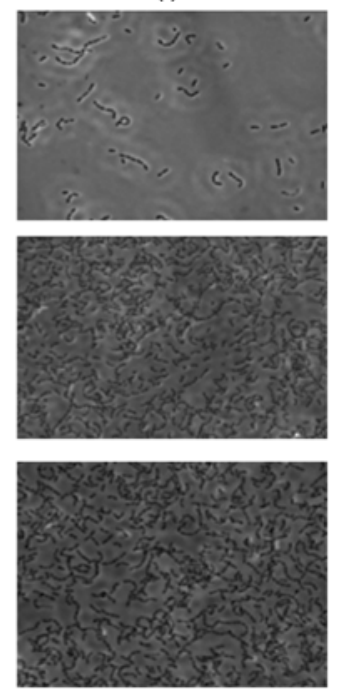

Fig. 2. Phase-contrast microscopic analysis of biofilm structure after $24 \mathrm{~h}$ in TSBG. (a) TX0014, (b) TX5454.01, (c) TX5454, (d) TX0006, (e) TX5453.01, (f) TX5453.
Dautle, M. P., Wilkinson, T. R. \& Gauderer, M. W. L. (2003). Isolation and identification of biofilm microorganisms from silicone gastrostomy devices. J Pediatr Surg 38, 216-220.

Dunman, P. M., Murphy, E., Haney, S. \& 7 other authors (2001). Transcription profiling-based identification of Staphylococcus aureus genes regulated by the agr and/or sarA loci. J Bacteriol 183, 7341-7353.

Engelbert, M., Mylonakis, E., Ausubel, F. M., Calderwood, S. B. \& Gilmore, M. S. (2004). Contribution of gelatinase, serine protease, and $f s r$ to the pathogenesis of Enterococcus faecalis endophthalmitis. Infect Immun 72, 3628-3633.

Hancock, L. E. \& Perego, M. (2004). The Enterococcus faecalis fsr two-component system controls biofilm development through production of gelatinase. J Bacteriol 186, 5629-5639.
Hufnagel, M., Koch, S., Creti, R., Baldassarri, L. \& Huebner, J. (2004). A putative sugar-binding transcriptional regulator in a novel gene locus in Enterococcus faecalis contributes to production of biofilm and prolonged bacteremia in mice. $J$ Infect Dis 189, 420-430.

Jacob, A. E. \& Hobbs, S. J. (1974). Conjugal transfer of plasmid-borne multiple antibiotic resistance in Streptococcus faecalis var. zymogenes. J Bacteriol 117, 360-372.

Joyanes, P., Pascual, A., Martinez-Martinez, L., Hevia, A. \& Perea, E. J. (1999). In vitro adherence of Enterococcus faecalis and Enterococcus faecium to plastic biomaterials. Clin Microbiol Infect 5, 382-386.

Keane, P. F., Bonner, M. C., Johnston, S. R., Zafar, A. \& Gorman, S. P. (1994).

Characterization of biofilm and encrustation on ureteric stents in vivo. Br J Urol 73, 687-691.
Kristich, C. J., Li, Y.-H., Cvitkovitch, D. G. \& Dunny, G. M. (2004). Esp-independent biofilm formation by Enterococcus faecalis. J Bacteriol 186, 154-163.

Mohamed, J. A., Singh, K. V., Huang, W., Teng, F. \& Murray, B. E. (2003). Influence of clinical origin and of various genes on biofilm formation by Enterococcus faecalis. In Program and Abstracts of the 43rd Annual Interscience Conference on Antimicrobial Agents and Chemotherapy (Chicago), abstract B-821, p. 52. Washington, DC: American Society for Microbiology.

Mohamed, J. A., Huang, W., Nallapareddy, S. R., Teng, F. \& Murray, B. E. (2004). Influence of origin of isolates, especially endocarditis isolates, and various genes on biofilm formation by Enterococcus faecalis. Infect Immun 72, 3658-3663. 
Mohamed, J. A., Teng, F., Nallapareddy, S. R. \& Murray, B. E. (2006). Pleiotrophic effects of 2 Enterococcus faecalis sagA-like genes, salA and $s a l B$, which encode proteins that are antigenic during human infection, on biofilm formation and binding to collagen type I and fibronectin. J Infect Dis 193, 231-240.

Murray, B. E. (1990). The life and times of the Enterococcus. Clin Microbiol Rev 3, 46-65.

Nakayama, J., Cao, Y., Horii, T., Sakuda, S., Akkermans, A. D. L., de Vos, W. M. \& Nagasawa, H. (2001). Gelatinase biosynthesis-activating pheromone, a peptide lactone that mediates a quorum sensing in Enterococcus faecalis. Mol Microbiol 41, 145-154.

Nallapareddy, S. R., Wenxiang, H., Weinstock, G. M. \& Murray, B. E. (2005). Molecular characterization of a widespread, pathogenic, and antibiotic resistance-receptive Enterococcus faecalis lineage and dissemination of its putative pathogenicity island. J Bacteriol 187 5709-5718.

Pillai, S. K., Sakoulas, G., Eliopoulos, G. M., Moellering, R. C., Jr, Murray, B. E. \& Inouye,

R. T. (2004). Effects of glucose on $f s r$-mediated biofilm formation in Enterococcus faecalis. J Infect Dis 190, 967-970.

Qin, X., Singh, K. V., Weinstock, G. M. \& Murray, B. E. (2000). Effects of Enterococcus faecalis $f s r$ genes on production of gelatinase and a serine protease and virulence. Infect Immun 68, 2579-2586.
Qin, X., Singh, K. V., Weinstock, G. M. \& Murray, B. E. (2001). Characterization of $f s r$, a regulator controlling expression of gelatinase and serine protease in Enterococcus faecalis OG1RF. J Bacteriol 183, 3372-3382.

Roberts, J. C., Singh, K. V., Okhuysen, P. C. \& Murray, B. E. (2004). Molecular epidemiology of the $f_{s} r$ locus and of gelatinase production among different subsets of Enterococcus faecalis isolates. J Clin Microbiol 42, 2317-2320.

Sandoe, J. A. T., Witherden, I. R., Cove, J. H., Heritage, J. \& Wilcox, M. H. (2003). Correlation between enterococcal biofilm formation in vitro and medical-device-related infection potential in vivo. J Med Microbiol 52, 547-550.

Seno, Y., Kariyama, R., Mitsuhata, R., Monden, K. \& Kumon, H. (2005). Clinical implications of biofilm formation by Enterococcus faecalis in the urinary tract. Acta Med Okayama 59, 79-87.

Singh, K. V., Qin, X., Weinstock, G. M. \& Murray, B. E. (1998). Generation and testing of mutants of Enterococcus faecalis in a mouse peritonitis model. J Infect Dis 178, 1416-1420.

Singh, K. V., Nallapareddy, S. R., Nannini, E. C. \& Murray, B. E. (2005). Fsr-independent production of protease(s) may explain the lack of attenuation of an Enterococcus faecalis $f_{s} r$ mutant versus a gelE-sprE mutant in induction of endocarditis. Infect Immun 73, 4888-4894.
Toledo-Arana, A., Valle, J., Solano, C. \& 7 other authors (2001). The enterococcal surface protein, Esp, is involved in Enterococcus faecalis biofilm formation. Appl Environ Microbiol 67, 4538-4545.

Trieu-Cuot, P., Carlier, C., Poyart-Salmeron, C. \& Courvalin, P. (1991). Shuttle vectors containing a multiple cloning site and a lac $Z \alpha$ gene for conjugal transfer of DNA from Escherichia coli to Gram-positive bacteria. Gene 102, 99-104.

Vuong, C., Saenz, H. L., Götz, F. \& Otto, M. (2000). Impact of the agr quorum-sensing system on adherence to polystyrene in Staphylococcus aureus. J Infect Dis 182, 1688-1693

Vuong, C., Gerke, C., Somerville, G. A. Fischer, E. R. \& Otto, M. (2003) Quorum-sensing control of biofilm factors in Staphylococcus epidermidis. J Infect Dis 188, 706-718.

Vuong, C., Kocianova, S., Yao, Y., Carmody, A. B. \& Otto, M. (2004). Increased colonization of indwelling medical devices by quorum-sensing mutants of Staphylococcus epidermidis in vivo. J Infect Dis $\mathbf{1 9 0}$, 1498-1505.

Zeng, J., Teng, F. \& Murray, B. E. (2005). Gelatinase is important for translocation of Enterococcus faecalis across polarized human enterocyte-like T84 cells. Infect Immun 73, 1606-1612. 